published findings can be found online (http://bjp.rcpsych.org/cgi/eletters/190/49/ s39).

Contrary to Cooke et al, the four-factor model clearly fits as well or better than a viable three-factor model. Moreover, our recent research indicates that the four first-order factors are explained by a cohesive superordinate factor (Neumann et al, 2006, 2007).

Cooke, D. J. \& Michie, C. (200I) Refining the construct of psychopathy: towards a hierarchical model. Psychological Assessment, 13, 17|-188.

Cooke, D. J., Michie, C. \& Skeem, J. (2007) Understanding the structure of the Psychopathy Checklist - Revised. An exploration of methodological confusion. British Journal of Psychiatry, 190 (suppl. 49), s39-s50.

Everitt, B. \& Dunn, G. (200I) Applied Multivariate Dato Analysis. Oxford University Press.

Neumann, C. S., Kosson, D. S., Forth, A. E., et al (2006) Factor structure of the Hare Psychopathy Checklist:Youth Version (PCL:YV) in incarcerated adolescents. Psychological Assessment, 18, 142-154.

Neumann, C. S., Hare, R. D. \& Newman, J. P. (2007) The super-ordinate nature of the Psychopathy ChecklistRevised. Journal of Personality Disorders, 2I, 102-117.

Skeem, J. L., Mulvey, E. P. \& Grisso, T. (2003)

Applicability of traditional and revised models of psychopathy to the Psychopathy Checklist: Screening Version. Psychological Assessment, 15, 4-55.

C. Neumann Department of Psychology,

University of North Texas, USA.

Email: csn000।@unt.edu

doi: 10.1192/bjp.191.4.357a

\section{Involuntary community treatment}

Swanson et al (2000) reanalysed the results of the North Carolina trial (Swartz et al, 1999) and their findings are becoming increasingly influential in current debates about mental health legislation in the UK. Our recent systematic review (Churchill et al, 2007), which included these articles, demonstrated that there was no robust evidence to indicate that community treatment orders are associated with either significant benefit or harm. The secondary analyses performed by Swanson et al are, we believe, misleading for two reasons.

First, based on everyone in the trial the intention-to-treat (ITT) effect of randomisation to an involuntary out-patient commitment (OPC) was of a modest and non-significant reduction in violence (risk difference of $4.5 \%$ ). This overall ITT effect of OPCs is a weighted average of the ITT effects in the two subgroups of participants defined by their post-randomisation management (those who received shortterm OPCs and those who eventually received long-term OPCs). These two subgroups would exist in the control arm had they been placed on OPCs. Assuming that there was no benefit in those who received the short-term OPCs (i.e. risk difference 0 ), the results of Swanson et al suggest that the reduction in violence in those with longterm OPCs would be $12.4 \%$. However, even if considered clinically significant, this finding would still not be statistically significant because the overall ITT effect was not significant (assuming a zero ITT effect in those receiving short-term OPCs implies that a test of the hypothesis concerning those receiving long-term OPCs is equivalent to the test for the overall ITT effect). The only way in which there could have been a beneficial effect in those receiving long-term OPCs is if the effects in those receiving short-term OPCs were actually detrimental (i.e. increased the rate of violence). It is improbable that they would be, and in policy terms it would be unacceptable to impose OPCs in the knowledge that they would cause harm to those in whom they are only applied for a short period.

Second, a post hoc comparison of the outcomes in groups defined by management decisions or patient behaviour following randomisation is potentially subject to selection effects (hidden confounding). That this is in fact the case is illustrated by the results of other subgroup analyses by the same research group (Swartz et al, 1999: Fig. 1). The group destined to be on long-term OPC have a better clinical outcome in the first 1-2 months. In other words there is evidence that the group destined to receive long-term OPCs have a favourable clinical profile before the OPC is renewed. We believe that it is likely that long-term OPCs will only be contemplated under certain circumstances, such as when the short-term OPC has apparently made a difference. Those who have intractable problems or in whom a short-term OPC has failed to make any change might not have their OPC renewed.

The investigators responsible for the North Carolina trial accomplished one of the most extraordinary trials ever performed and as such deserve enormous praise. However, the results described in these and similar secondary analyses are, we believe, flawed and misleading, and should not be taken as evidence for a beneficial effect of OPC. We made a similar point (Szmukler \& Hotopf, 2001) following the publication of the original trial. The trial data are best interpreted using the main ITT analyses, which show no evidence of benefit or harm.

Churchill, R., Owen, G., Singh, S., et al (2007) International Experiences of Using Community Treatment Orders. Department of Health.

Swanson, J. W., Swartz, M. S., Wagner, H. R., et al (2000) Involuntary out-patient commitment and reduction of violent behaviour in persons with severe mental illness. British Journal of Psychiatry, I76, 324-331.

Swartz, M. S., Swanson, J.W., Wagner, H. R., et al (1999) Can involuntary outpatient commitment reduce hospital recidivism? Findings from a randomized trial with severely mentally ill individuals. American Journal of Psychiatry, 156, 1968-1975.

Szmukler, G. \& Hotopf, M. (200I) Effectiveness of involuntary outpatient commitment. American Journal of Psychiatry, 158, 653-654.

M. Hotopf Institute of Psychiatry, King's College London, UK. Email: m.hotopf@iop.kcl.ac.uk

G. Dunn University of Manchester, UK

G. Owen, R. Churchill Institute of Psychiatry, King's College London, UK

doi: 10.1192/bjp.191.4.358

Authors'reply: Hotopf et al make essentially the same point that we stated in the article '. . . the study found no significant difference in the prospective rate of violence between the two randomly assigned groups: $32.3 \%$ in the OPC group $v$. $36.8 \%$ in the control group (Fisher's exact test, one-tailed: $\quad P=0.292$; two-tailed: $P=0.567)$ ' (Swanson et al, 2000).

Critics of OPC policy might wish we had left it at that, but straightforward analysis of randomised controlled trials does not tell the whole story. In this case it excluded people with a documented history of serious violence $(n=64)$, since the court did not permit us to randomise these to the control group. However, variability in the real-world application of OPC allowed us to examine whether longer periods of court-ordered treatment were associated with lower rates of violence over the study year. They were.

Hotopf et al are rightly concerned about the possibility of favourable selection bias, but we think this is an unlikely explanation for our findings. Indeed, people with a history of treatment non-adherence were more than twice as likely to receive an extended period of OPC (40.0 v. 18.75\%). If anything, this should have stacked the deck against finding an effect for long-term OPC. 
Hotopf et al recalculated the post-randomisation effect for longer-term OPC in what they refer to as our ITT sample, rather than the sample we actually used. They say the effect is not significant but their calculation excludes the historically violent subgroup.

For hospital outcomes, unlike violence, we obtained follow-up information on the entire ITT sample through admission records. Here we found a statistically significant experimental result. For any month during the study year, the randomly assigned OPC group had a lower risk of readmission than the control group $(\mathrm{OR}=0.64, P<0.01)$. Hotopf et al do not mention this finding.

About one-third of the OPC group had their court orders expire very early in the study - during the first or second month and more of these individuals were rehospitalised than those remaining on OPC, which explains the early separation of the lines in the figures from Swartz et al (1999).

Swanson, J. W., Swartz, M. S., Wagner, H. R., et al (2000) Involuntary out-patient commitment and reduction of violent behaviour in persons with severe mental illness. British Journal of Psychiatry, 176, 324-331.

Swartz, M. S., Swanson, J.W., Wagner, H. R., et al (1999) Can involuntary outpatient commitment reduce hospital recidivism? Findings from a randomized trial with severely mentally ill individuals. American journal of Psychiatry, 156, 1968-1975.

J. Swanson Department of Psychiatry and Behavioral Sciences, Duke University School of Medicine, Durham, NC 277I0, USA. Email: jeffrey.swanson@duke.edu

M. Swartz Division of Social and Community Psychiatry, Department of Psychiatry and Behavioral Sciences, Duke University School of Medicine,

Durham, USA

doi: 10.1192/bjp.191.4.358a

\section{Psychosocial interventions for self-harm}

Crawford et al (2007) conclude that the results of their meta-analysis 'do not provide evidence that additional psychosocial interventions following self-harm have a marked effect on the likelihood of subsequent suicide'. This conclusion is far too bold considering the weaknesses inherent in the analytical approach employed. In my opinion Crawford et al have not allowed adequate weight for several methodological problems, the most prominent being the rationale for including studies in the analysis.
They acknowledge the 'lack of statistical power' in the meta-analysis but offer a definitive and sweeping conclusion.

The lack of statistical power is only one reason not to conduct the meta-analysis. The central rationale for clustering the included studies is seriously flawed. Not only have they mixed simple interventions and treatments, the target populations range from latency-age children (some as young as 12 years) to older adults ( $>50$ years), intervention methods and theoretical orientations vary considerably (employing individual, group, case-management and home-based care), samples include those making suicide attempts as well as those engaging in self-harm (non-suicidal) behaviour, and they have also included studies that employed questionable intervention or treatment protocols for suicidality. A review of the intervention and treatment protocols of the studies included reveals wide variability in the nature, oversight and fidelity of the services being offered. I have serious concerns about at least 8 of the 19 study protocols. Some of the interventions cannot realistically be described as appropriate for suicidality, at least from the perspective that they have a serious chance of reducing subsequent pathology of suicide attempts, much less actual deaths. For example, Harrington et al (1998) employed four home visits by a social worker. Similarly, Guthrie et al (2001) included four sessions delivered in the patient's home. Cedereke et al (2002) explored the utility of random telephone interventions and Clarke et al (2002) included 'management enhanced by nurseled case management'. As these examples illustrate, not all psychosocial interventions are the same, something Crawford et al (2007) failed to clarify in their article. Why would we expect that a meta-analysis of randomised trials of interventions or treatments that are this broadly disparate (with samples equally disparate) would actually provide evidence of effective reduction of subsequent suicides?

Meta-analyses have become increasingly popular and increasingly misleading in their findings. Prior to inclusion in a meta-analysis of intervention or treatment outcome, I would suggest a thorough review of the intervention/treatment approach and related fidelity. Only those studies meeting strict and predefined criteria should be included. When considering strategies for including and clustering treatment studies for meta-analysis, it is particularly important to consider the targeted problem or disorder. Many, if not most problems targeted by psychosocial interventions and treatments are recurrent, persistent and potentially chronic in nature. Hence, the need for careful scrutiny of studies included.

Compounding the problems noted above, the follow-up periods for all of the studies included by Crawford et al ranged from 6 to 12 months. The efficacy of treatment or interventions for suicide will only be known after 5,10 or 20 years. In shorterterm studies even if the results did show a reduction in subsequent suicides, we would not know whether the interventions or treatments were 'delaying' suicide or actually preventing it without longitudinal data.

There are many other factors that need to be scrutinised prior to inclusion of studies in a meta-analysis (e.g. sample size, categorisation of attempt status and suicide intent, fidelity/oversight of intervention or treatment) but space does not allow a full discussion. The point is that identifying appropriate inclusion criteria for such a study is a complex process which is far more complicated than simply taking all randomised controlled trials.

The definitive nature of the conclusion offered by Crawford et al belies the current state of the science in this area. In an age when legislators and funding agencies rely on science for direction, studies like this one generate ill-informed conclusions on what interventions, treatments and approaches to suicide prevention offer the most promise. Many readers will sadly and mistakenly carry away the message that psychosocial interventions offer no promise to reduce suicide rates.

Cedereke, M., Monti, K. \& Ojehagen, A. (2002) Telephone contact with patients in the year after a suicide attempt: does it affect treatment attendance and outcome? European Psychiatry, 17, 82-91.

Clarke, T., Baker, P., Watts, C. J., et al (2002) Selfharm in adults: a randomized controlled trial of nurseled management versus routine care only. Journal of Mental Health, II, 167-176.

Crawford, M. J., Thomas, O., Khan, N., et al (2007) Psychosocial interventions following self-harm: systematic review of their efficacy in preventing suicide. British Journal of Psychiatry, 190, II-17.

Guthrie, E., Kapur, N., Mackway-Jones, K., et al (200I) Randomized controlled trial of brief psychological intervention after deliberate self-poisoning. BM), 323, 135-138.

Harrington, R., Kerfoot, M., Dyer, E., et al (1998) Randomized trial of a home-based intervention for children who have deliberately poisoned themselves. 